\title{
CP properties of symmetry-constrained two-Higgs-doublet models
}

\author{
P.M. Ferreira, ${ }^{(1,2)}$ \\ M. Maniatis, ${ }^{(3)}$ \\ O. Nachtmann, ${ }^{(3)}$ \\ and João P. Silva ${ }^{(1,4)}$ \\ (1) Instituto Superior de Engenharia de Lisboa \\ 1959-007 Lisboa, Portugal \\ (2) Centro de Física Teórica e Computacional, Universidade de Lisboa \\ 1649-003 Lisboa, Portugal \\ (3) Institut für Theoretische Physik \\ Philosophenweg 16, 69120 Heidelberg, Germany \\ (4) Centro de Física Teórica de Partículas, Instituto Superior Técnico \\ 1049-001 Lisboa, Portugal
}

\begin{abstract}
The two-Higgs-doublet model can be constrained by imposing Higgs-family symmetries and/or generalized CP symmetries. It is known that there are only six independent classes of such symmetry-constrained models. We study the CP properties of all cases in the bilinear formalism. An exact symmetry implies CP conservation. We show that soft breaking of the symmetry can lead to spontaneous $\mathrm{CP}$ violation $(\mathrm{CPV})$ in three of the classes.
\end{abstract}

\section{INTRODUCTION AND NOTATION}

Experiments will soon probe the electroweak symmetry breaking (EWSB) mechanism. This is achieved in the Standard Model (SM) with one Higgs doublet. A very simple extension involves two Higgs-field doublets $\varphi_{1}$ and $\varphi_{2}$, required also by supersymmetry. One particular feature of this model that makes it very popular is the possibility that it has vacua which spontaneously break the CP symmetry. Indeed this was the main reason why Lee introduced the two-Higgs-doublet model (THDM) in 1973 [1. In fact, it is known that the amount of CP violation that the Standard Model displays is insufficient to explain the observed matter-antimatter asymmetry in the universe. As such, models wherein CP violation arises from other mechanisms are in demand, and the THDM is the simplest such model. We will investigate whether CP is preserved in the potential, both before and after EWSB. We will consider all possible symmetry-constrained THDM potentials, both with exact and softly broken symmetries. And we will be able to conduct this study in an analytical manner, for most cases avoiding direct calculation of the fields' vacuum expectation values.

The most general potential of the THDM may be written in terms of fields as [2, 3]

$$
\begin{aligned}
V= & m_{11}^{2}\left(\varphi_{1}^{\dagger} \varphi_{1}\right)+m_{22}^{2}\left(\varphi_{2}^{\dagger} \varphi_{2}\right)-m_{12}^{2}\left(\varphi_{1}^{\dagger} \varphi_{2}\right)-\left(m_{12}^{2}\right)^{*}\left(\varphi_{2}^{\dagger} \varphi_{1}\right) \\
& +\frac{1}{2} \lambda_{1}\left(\varphi_{1}^{\dagger} \varphi_{1}\right)^{2}+\frac{1}{2} \lambda_{2}\left(\varphi_{2}^{\dagger} \varphi_{2}\right)^{2}+\lambda_{3}\left(\varphi_{1}^{\dagger} \varphi_{1}\right)\left(\varphi_{2}^{\dagger} \varphi_{2}\right) \\
& +\lambda_{4}\left(\varphi_{1}^{\dagger} \varphi_{2}\right)\left(\varphi_{2}^{\dagger} \varphi_{1}\right)+\frac{1}{2}\left[\lambda_{5}\left(\varphi_{1}^{\dagger} \varphi_{2}\right)^{2}+\lambda_{5}^{*}\left(\varphi_{2}^{\dagger} \varphi_{1}\right)^{2}\right] \\
& +\left[\lambda_{6}\left(\varphi_{1}^{\dagger} \varphi_{2}\right)+\lambda_{6}^{*}\left(\varphi_{2}^{\dagger} \varphi_{1}\right)\right]\left(\varphi_{1}^{\dagger} \varphi_{1}\right)+\left[\lambda_{7}\left(\varphi_{1}^{\dagger} \varphi_{2}\right)+\lambda_{7}^{*}\left(\varphi_{2}^{\dagger} \varphi_{1}\right)\right]\left(\varphi_{2}^{\dagger} \varphi_{2}\right),
\end{aligned}
$$

with $m_{11}^{2}, m_{22}^{2}, \lambda_{1,2,3,4}$ real and $m_{12}^{2}, \lambda_{5,6,7}$ complex. To study the properties of the Higgs potential, it is oftentimes easier to write it in terms of field bilinears [4, 15]. In [5, 8, 9] a one-to-one correspondence of gauge invariant expressions with bilinears with a simple geometric interpretation was revealed. In this approach the bilinears form a Minkowski type four vector. We follow the notation of the Heidelberg group [5, 8 , and write $V$ as

$$
V=\tilde{\mathbf{K}}^{\mathrm{T}} \tilde{\boldsymbol{\xi}}+\tilde{\mathbf{K}}^{\mathrm{T}} \tilde{E} \tilde{\mathbf{K}}
$$

with

$$
\tilde{\mathbf{K}}=\left(\begin{array}{c}
K_{0} \\
\mathbf{K}
\end{array}\right), \quad K_{0}=\varphi_{1}^{\dagger} \varphi_{1}+\varphi_{2}^{\dagger} \varphi_{2}, \quad \mathbf{K}=\left(\begin{array}{c}
\varphi_{1}^{\dagger} \varphi_{2}+\varphi_{2}^{\dagger} \varphi_{1} \\
i \varphi_{2}^{\dagger} \varphi_{1}-i \varphi_{1}^{\dagger} \varphi_{2} \\
\varphi_{1}^{\dagger} \varphi_{1}-\varphi_{2}^{\dagger} \varphi_{2}
\end{array}\right)
$$


In $1.2 \tilde{\xi}$ and $\tilde{E}$ contain the parameters, which are all real:

$$
\tilde{\boldsymbol{\xi}}=\left(\begin{array}{c}
\xi_{0} \\
\boldsymbol{\xi}
\end{array}\right), \quad \tilde{E}=\left(\begin{array}{cc}
\eta_{00} & \boldsymbol{\eta}^{\mathrm{T}} \\
\boldsymbol{\eta} & E
\end{array}\right)
$$

with $E=E^{\mathrm{T}}$ a $3 \times 3$ matrix. Expressing $\tilde{\boldsymbol{\xi}}$ and $\tilde{\mathbf{E}}$ in terms of the parameters of (1.1), we find

$$
\begin{gathered}
\xi_{0}=\frac{1}{2}\left(m_{11}^{2}+m_{22}^{2}\right), \quad \boldsymbol{\xi}=\frac{1}{2}\left(\begin{array}{c}
-2 \operatorname{Re}\left(m_{12}^{2}\right) \\
2 \operatorname{Im}\left(m_{12}^{2}\right) \\
m_{11}^{2}-m_{22}^{2}
\end{array}\right), \\
\eta_{00}=\frac{1}{8}\left(\lambda_{1}+\lambda_{2}\right)+\frac{1}{4} \lambda_{3}, \quad \boldsymbol{\eta}=\frac{1}{4}\left(\begin{array}{c}
\operatorname{Re}\left(\lambda_{6}+\lambda_{7}\right) \\
-\operatorname{Im}\left(\lambda_{6}+\lambda_{7}\right) \\
\frac{1}{2}\left(\lambda_{1}-\lambda_{2}\right)
\end{array}\right), \\
E=\frac{1}{4}\left(\begin{array}{ccc}
\lambda_{4}+\operatorname{Re}\left(\lambda_{5}\right) & -\operatorname{Im}\left(\lambda_{5}\right) & \operatorname{Re}\left(\lambda_{6}-\lambda_{7}\right) \\
-\operatorname{Im}\left(\lambda_{5}\right) & \lambda_{4}-\operatorname{Re}\left(\lambda_{5}\right) & -\operatorname{Im}\left(\lambda_{6}-\lambda_{7}\right) \\
\operatorname{Re}\left(\lambda_{6}-\lambda_{7}\right) & -\operatorname{Im}\left(\lambda_{6}-\lambda_{7}\right) & \frac{1}{2}\left(\lambda_{1}+\lambda_{2}\right)-\lambda_{3}
\end{array}\right),
\end{gathered}
$$

When using bilinears one must only take care that, by construction, they are restricted by

$$
K_{0} \geq 0, \text { and } K_{0}^{2}-\mathbf{K}^{2} \geq 0
$$

The fields $\varphi_{1}$ and $\varphi_{2}$ are not physical, only their mass eigenstates will be physical. Thus, we can choose for general discussions, for instance of symmetries and their breaking, any linear combination thereof that doesn't change the kinetic terms. The physics will have to remain the same for these basis changes, i.e. unitary transformations of the form

$$
\left(\begin{array}{l}
\varphi_{1} \\
\varphi_{2}
\end{array}\right) \rightarrow\left(\begin{array}{l}
\varphi_{1}^{\prime} \\
\varphi_{2}^{\prime}
\end{array}\right)=U\left(\begin{array}{l}
\varphi_{1} \\
\varphi_{2}
\end{array}\right)
$$

with $U=\left(U_{i j}\right) \in U(2)$. In bilinear language, this corresponds to a $S O(3)$ rotation in $K$ space, given by

$$
\begin{aligned}
& K_{0} \rightarrow K_{0}^{\prime}=K_{0}, \\
& \mathbf{K} \rightarrow \mathbf{K}^{\prime}=R(U) \mathbf{K} .
\end{aligned}
$$

Here $R(U)$ is obtained from

$$
U^{\dagger} \sigma^{a} U=R_{a b}(U) \sigma^{b}
$$

This freedom of choosing a basis may help to simplify many calculations, but it also may mask the symmetry of a potential. Thus, basis-invariant methods are advantageous in many problems.

CP properties of the THDM Higgs potential were studied extensively in the literature in a basis-independent way [9, 11, 12, 14, 16, 22. Here we focus on a study of CP properties in terms of the bilinears. As shown in [11, the standard CP transformation of the Higgs doublets

$$
\varphi_{i}(x) \rightarrow \varphi_{i}^{*}\left(x^{\prime}\right), \quad i=1,2
$$

corresponds in $K$ space to

$$
\begin{aligned}
K_{0}(x) & \rightarrow K_{0}\left(x^{\prime}\right) \\
\mathbf{K}(x) & \rightarrow \operatorname{diag}(1,-1,1) \mathbf{K}\left(x^{\prime}\right) .
\end{aligned}
$$

The parity transformation flips the sign of the spatial components in $x \rightarrow x^{\prime}$. Geometrically, a standard CP transformation is a reflection of the $\mathbf{K}$ vector on the 1-3 plane in a certain basis in addition to the parity transformation of the argument. 
We shall also consider generalized CP transformations (GCPs) as discussed already in [16, 17, 23, 28, where the standard CP transformation is accompanied by a mixing of the Higgs fields:

$$
\varphi_{i}(x) \rightarrow X_{i j} \varphi_{j}^{*}\left(x^{\prime}\right), \quad i, j=1,2,
$$

with $X=\left(X_{i j}\right) \in U(2)$. In a suitable basis for the fields the matrix $X$ in 1.14 ) can always be brought to the form [27]

$$
X=\left(\begin{array}{cc}
\cos (\theta) & \sin (\theta) \\
-\sin (\theta) & \cos (\theta)
\end{array}\right), \quad \text { with } 0 \leq \theta \leq \pi / 2 .
$$

These transformations were classified in [11, 29]. Following here the notation in [29] we have

$$
\begin{array}{lll}
\mathrm{CP} 1 & \text { if } & \theta=0, \\
\mathrm{CP} 2 & \text { if } & \theta=\pi / 2, \\
\mathrm{CP} 3 & \text { if } & 0<\theta<\pi / 2 .
\end{array}
$$

In $K$ space the GCP transformations 1.14 correspond to the improper rotations

$$
\mathbf{K}(x) \rightarrow \bar{R} \mathbf{K}\left(x^{\prime}\right)
$$

with

$$
\bar{R}=\left(\begin{array}{ccc}
\cos (2 \theta) & 0 & -\sin (2 \theta) \\
0 & -1 & 0 \\
\sin (2 \theta) & 0 & \cos (2 \theta)
\end{array}\right), \quad 0 \leq \theta \leq \pi / 2
$$

in the specific basis where $X$ has the form (1.15). From this representation we identify CP1 as reflections on planes and CP2 as the point reflection at the origin in addition to the parity transformation of the argument.

We emphasize that the symmetries CP1, CP2, and CP3 are independent symmetries, which may be imposed on the Lagrangian. However, considering only the THDM scalar sector, invariance under CP2 or CP3 implies invariance under CP1, as shown in [29]. This will also be seen directly from the discussion in section 2 below. Furthermore, considering only the scalar sector, no Higgs basis is preferred and, a priori, any GCP transformation of type CP1 is as good as any other to be called the CP transformation. In the sequel, we will, therefore, simply talk about $C P$ symmetry, CP conservation, and CP violation and drop the notation generalized.

In the following, we will use basis-invariant quantities, that is, quantities invariant under the transformations (1.9), respectively in $K$ space, the rotations (1.10). We shall also work in bases which are clearly singled out by the problem at hand. This can be the basis implied by (1.15), respectively (1.18), or the basis where $E$ is diagonal. Note that the eigenvalues of $E$ and the angle $\theta$ in (1.15), (1.18) are basis independent quantities. For the eigenvalues of $E$ this is so by construction. To see this for the angle $\theta$ we note that by a basis transformation (1.9) $X$ in (1.14) is replaced by

$$
X^{\prime}=U X U^{\mathrm{T}} .
$$

This implies

$$
X^{\prime} X^{*}=U X X^{*} U^{\dagger}
$$

It is easy to see from (1.15) that $\exp ( \pm 2 i \theta)$ are the eigenvalues of $X X^{*}$ in the standard form. But using 1.20 we see that these are then also the eigenvalues of $X X^{*}$ in any basis, that is, $\theta$ is basis independent.

One can construct basis invariant quantities which measure explicit CP violation at the level of the THDM Lagrangian, that is, before EWSB [9, 11, 18, 22. In terms of the potential parameters (1.5)-(1.7) a convenient set of such quantities (see theorem 3 of [11]) is given by [40]

$$
\begin{aligned}
I_{1} & =(\boldsymbol{\xi} \times \boldsymbol{\eta})^{\mathrm{T}} E \boldsymbol{\xi}, \\
I_{2} & =(\boldsymbol{\xi} \times \boldsymbol{\eta})^{\mathrm{T}} E \boldsymbol{\eta}, \\
I_{3} & =(\boldsymbol{\xi} \times(E \boldsymbol{\xi}))^{\mathrm{T}} E^{2} \boldsymbol{\xi}, \\
I_{4} & =(\boldsymbol{\eta} \times(E \boldsymbol{\eta}))^{\mathrm{T}} E^{2} \boldsymbol{\eta} .
\end{aligned}
$$


The theory is $\mathrm{CP}$ conserving at the Lagrangian level, meaning that it allows for at least one CP transformation as a symmetry, if and only if all $I^{\prime} s$ vanish.

Suppose now that the Higgs potential is indeed invariant under at least one CP transformation, that is, all $I$ 's vanish. Then, there is at least one $\mathrm{CP}$ invariance respected simultaneously at the Lagrangian level and by the vacuum if also the three following basis-invariant quantities vanish [41,

$$
\begin{aligned}
J_{1} & =(\boldsymbol{\xi} \times \boldsymbol{\eta})^{\mathrm{T}}\langle\mathbf{K}\rangle, \\
J_{2} & =(\boldsymbol{\xi} \times(E \boldsymbol{\xi}))^{\mathrm{T}}\langle\mathbf{K}\rangle, \\
J_{3} & =(\boldsymbol{\eta} \times(E \boldsymbol{\eta}))^{\mathrm{T}}\langle\mathbf{K}\rangle .
\end{aligned}
$$

See theorem 6 of [1] and [16, 17]. Here $\langle\mathbf{K}\rangle$ denotes the $\mathbf{K}$ vector formed by the vacuum expectation values $\left\langle\varphi_{i}\right\rangle$ of the fields according to (1.3). Let us emphasize that in the case of more than one CP invariance of the theory the statement is that at least one $\mathrm{CP}$ invariance persists after EWSB if $J_{1}=J_{2}=J_{3}=0$. In this case we call the Higgs potential spontaneously $\mathrm{CP}$ conserving.

To summarize: the conditions $\sqrt{1.21}-(1.24), \sqrt{1.25}-(1.27)$ are relevant to study the theoretical GCP properties of any given model: i) if all $I^{\prime} s$ and $J^{\prime} s$ vanish, then there is at least one CP transformation which is conserved at the Lagrangian level and also after EWSB. Certainly, this CP symmetry would then be considered as the CP symmetry of the theory. This means that any CP violating experiment, such as a particle/antiparticle decay asymmetry, will yield null results. In the following we call this the no $C P$ violating case - but note the caveat below. ii) If all $I^{\prime} s$ vanish and at least one $J$ does not, then there is spontaneous CP violation. iii) If at least one $I$ differs from zero, then the theory has explicit $\mathrm{CP}$ violation. We caution the reader regarding the following fact. A theory may have more than one GCP transformation as a symmetry before EWSB. In this case the vanishing of all $I^{\prime} s$ and $J^{\prime} s$ means that, out of these GCP symmetries, at least one is preserved by the vacuum. As such, it will be possible to define particles (scalars and pseudoscalars) which are CP eigenstates, which affects the way they couple to the $Z$ boson, for example. However this does not imply that all GCP symmetries are likewise preserved. One such example is the model discussed in [30] which has, at the Lagrangian level, four GCP symmetries. Of these two are broken by the vacuum-expectation value, while two other ones are preserved.

The $J$ quantities involve the vacuum-expectation-values (vevs) of the fields. If the theory has a neutral vacuum we may write without loss of generality

$$
\begin{aligned}
& \left\langle\varphi_{1}\right\rangle=\left(\begin{array}{c}
0 \\
v_{1}
\end{array}\right) \\
& \left\langle\varphi_{2}\right\rangle=\left(\begin{array}{c}
0 \\
v_{2} e^{i \zeta}
\end{array}\right)
\end{aligned}
$$

where $v_{1}$ and $v_{2}$ are real and the standard Higgs vacuum-expectation value is $v_{0}=\sqrt{2\left(v_{1}^{2}+v_{2}^{2}\right)} \approx 246 \mathrm{GeV}$. Inserting 1.28 in 1.3 we find

$$
\langle\tilde{\mathbf{K}}\rangle=\left(\begin{array}{c}
\left\langle K_{0}\right\rangle \\
\langle\mathbf{K}\rangle
\end{array}\right)=\left(\begin{array}{c}
v_{0}^{2} / 2 \\
2 v_{1} v_{2} \cos \zeta \\
2 v_{1} v_{2} \sin \zeta \\
v_{1}^{2}-v_{2}^{2}
\end{array}\right)
$$

The vacuum-expectation values are determined by the stationarity conditions. In the field language we need to set the gradient of the potential to zero. In the bilinear formalism, we must find the solutions of:

$$
(\tilde{E}-u \tilde{g}) \tilde{\mathbf{K}}=-\frac{1}{2} \tilde{\xi}, \quad K_{0}^{2}-\mathbf{K}^{2}=0, \quad K_{0}>0
$$

see section 5 of [8]. Here a Lagrange multiplier $u$ is introduced in order to respect the second constraint of $(1.30)$ and $\tilde{g}=\operatorname{diag}(1,-1,-1,-1)$. We have five equations in five variables $K_{0}, K_{1}, K_{2}, K_{3}$, u, and one inequality. Of course, one has to make sure to pick out from the stationary points the absolute minimum of the potential as the vacuum solution.

Note that here we focus on the CP properties of Higgs potentials. For a study of their stability, electroweak symmetry breaking, and of the solution of $[1.30$ corresponding to the global minimum we refer to [8]. There, necessary and sufficient conditions for physically required properties of the potential are given in terms of the bilinear formalism. We can add, however, that for all models discussed below - with both exact and softly broken symmetries - we were able to find numerical examples of viable minima. Thus, all classes of models below are of physical interest. 


\section{SYMMETRIES OF THE THDM}

The parameters in 1.1) may be reduced by imposing some symmetry of the type $\varphi_{i}(x) \rightarrow S_{i j} \varphi_{j}(x)$, or $\varphi_{i}(x) \rightarrow X_{i j} \varphi_{j}^{*}\left(x^{\prime}\right)$, where $S$ and $X$ belong to $U(2)$. The former are known as family symmetries, the latter as generalized CP symmetries [23 28]. Recently it has been shown that applying the symmetries with any possible choices for $S$ and $X$ leads only to six classes of scalar potentials [13, 29].

Let us begin with the family symmetries.

- The $Z_{2}$ transformation [31, 32] is defined as

$$
\left(\begin{array}{l}
\varphi_{1} \\
\varphi_{2}
\end{array}\right) \rightarrow\left(\begin{array}{cc}
1 & 0 \\
0 & -1
\end{array}\right)\left(\begin{array}{l}
\varphi_{1} \\
\varphi_{2}
\end{array}\right)
$$

Using 1.3 we find the corresponding transformation in $K$ space:

$$
\left(\begin{array}{l}
K_{1} \\
K_{2} \\
K_{3}
\end{array}\right) \rightarrow\left(\begin{array}{ccc}
-1 & 0 & 0 \\
0 & -1 & 0 \\
0 & 0 & 1
\end{array}\right)\left(\begin{array}{l}
K_{1} \\
K_{2} \\
K_{3}
\end{array}\right)
$$

with $K_{0}$ unchanged. That is, in $K$ space $Z_{2}$ is a rotation by $\pi$ around the third axis. Using (1.2) and (1.4) we see that $Z_{2}$ is a symmetry of the potential $V$ if and only if

$$
\boldsymbol{\xi}=\left(\begin{array}{c}
0 \\
0 \\
\xi_{3}
\end{array}\right), \quad \boldsymbol{\eta}=\left(\begin{array}{c}
0 \\
0 \\
\eta_{3}
\end{array}\right), \quad E=\left(\begin{array}{ccc}
\eta_{11} & \eta_{12} & 0 \\
\eta_{12} & \eta_{22} & 0 \\
0 & 0 & \eta_{33}
\end{array}\right) .
$$

Eventually, by a change of basis, we can achieve the following form for the parameters of the Higgs potential

$$
Z_{2}: \quad \boldsymbol{\xi}=\left(\begin{array}{c}
0 \\
0 \\
\xi_{3}
\end{array}\right), \quad \boldsymbol{\eta}=\left(\begin{array}{c}
0 \\
0 \\
\eta_{3}
\end{array}\right), \quad E=\left(\begin{array}{ccc}
\mu_{1} & 0 & 0 \\
0 & \mu_{2} & 0 \\
0 & 0 & \mu_{3}
\end{array}\right) .
$$

A basis-invariant characterisation of this symmetry class is that both $\boldsymbol{\xi}$ and $\boldsymbol{\eta}$ are proportional to one and the same eigenvector of $E$.

- The Peccei-Quinn transformation $U(1)$ is defined as 33 35]

$$
\left(\begin{array}{l}
\varphi_{1} \\
\varphi_{2}
\end{array}\right) \rightarrow\left(\begin{array}{cc}
e^{-i \alpha} & 0 \\
0 & e^{i \alpha}
\end{array}\right)\left(\begin{array}{l}
\varphi_{1} \\
\varphi_{2}
\end{array}\right), \quad \text { with } 0 \leq \alpha<\pi
$$

In $K$ space we get via 1.3

$$
\left(\begin{array}{l}
K_{1} \\
K_{2} \\
K_{3}
\end{array}\right) \rightarrow\left(\begin{array}{ccc}
\cos (2 \alpha) & -\sin (2 \alpha) & 0 \\
\sin (2 \alpha) & \cos (2 \alpha) & 0 \\
0 & 0 & 1
\end{array}\right)\left(\begin{array}{l}
K_{1} \\
K_{2} \\
K_{3}
\end{array}\right)
$$

That is, the $U(1)$ transformations correspond to rotations around the third axis.

The potential $V$ is Peccei-Quinn symmetric if and only if there is a basis for which the parameters are

$$
U(1): \quad \boldsymbol{\xi}=\left(\begin{array}{c}
0 \\
0 \\
\xi_{3}
\end{array}\right), \quad \boldsymbol{\eta}=\left(\begin{array}{c}
0 \\
0 \\
\eta_{3}
\end{array}\right), \quad E=\left(\begin{array}{ccc}
\mu_{1} & 0 & 0 \\
0 & \mu_{1} & 0 \\
0 & 0 & \mu_{3}
\end{array}\right),
$$

as we easily see, combining (1.2), 1.4 , and 2.6). Here a basis independent formulation is that two eigenvalues of $E$ must be degenerate and both vectors $\boldsymbol{\xi}$ and $\boldsymbol{\eta}$ must be proportional to the eigenvector corresponding to the remaining third eigenvalue of $E$. 
- The $U(2)$ transformations in field space are given by

$$
\varphi_{i}(x) \rightarrow S_{i j} \varphi_{j}(x), \quad i=1,2,
$$

with $S=\left(S_{i j}\right) \in U(2)$. In $K$ space this corresponds to

$$
\mathbf{K} \rightarrow R \mathbf{K}, \quad \text { with } R \in S O(3) .
$$

The Higgs potential, invariant under all such transformations, has parameters

$$
U(2): \quad \boldsymbol{\xi}=\left(\begin{array}{l}
0 \\
0 \\
0
\end{array}\right), \quad \boldsymbol{\eta}=\left(\begin{array}{l}
0 \\
0 \\
0
\end{array}\right), \quad E=\left(\begin{array}{ccc}
\mu_{1} & 0 & 0 \\
0 & \mu_{1} & 0 \\
0 & 0 & \mu_{1}
\end{array}\right) .
$$

Formulated in a basis-independent way we have $\boldsymbol{\xi}=0$ and $\boldsymbol{\eta}=0$ and $E$ proportional to the unit matrix.

Now we come to the models which have generalized CP symmetries of the different types 1.16.

- The CP1 transformations with standard form $\theta=0$ in 1.15) correspond in $K$ space to reflections on planes in addition to the parity transformation of the argument. By a suitable basis change, any CP1 reflection can be rotated to occur on the 1-3 plane, as given in (1.13). The parameters of a Higgs potential, invariant under a $\mathrm{CP} 1$ transformation, can, with a suitable basis change, always be brought to the form

$$
\mathrm{CP} 1: \quad \boldsymbol{\xi}=\left(\begin{array}{c}
\xi_{1} \\
0 \\
\xi_{3}
\end{array}\right), \quad \boldsymbol{\eta}=\left(\begin{array}{c}
\eta_{1} \\
0 \\
\eta_{3}
\end{array}\right), \quad E=\left(\begin{array}{ccc}
\mu_{1} & 0 & 0 \\
0 & \mu_{2} & 0 \\
0 & 0 & \mu_{3}
\end{array}\right) .
$$

Formulated in a basis-independent way this means that $\boldsymbol{\xi}$ and $\boldsymbol{\eta}$ must be orthogonal to one and the same eigenvector of $E$.

- Generalized CP transformations of type $\mathrm{CP} 2$ correspond in $K$ space to the point reflection at the origin in addition to the parity transformation of the argument,

$$
\mathbf{K}(x) \rightarrow-\mathbb{1}_{3} \mathbf{K}\left(x^{\prime}\right) .
$$

Therefore, the Higgs potential parameters with this symmetry are, in a basis where $E$ is diagonal [11, 21],

$$
\mathrm{CP} 2: \quad \boldsymbol{\xi}=\left(\begin{array}{l}
0 \\
0 \\
0
\end{array}\right), \quad \boldsymbol{\eta}=\left(\begin{array}{l}
0 \\
0 \\
0
\end{array}\right), \quad E=\left(\begin{array}{ccc}
\mu_{1} & 0 & 0 \\
0 & \mu_{2} & 0 \\
0 & 0 & \mu_{3}
\end{array}\right) \text {. }
$$

The basis-independent requirement is here $\boldsymbol{\xi}=0$ and $\boldsymbol{\eta}=0$.

- Finally, the generalized CP transformations of type CP3 correspond in $K$ space to improper rotations $\bar{R}$ in 1.18 ) with $0<\theta<\pi / 2$ in addition to $x \rightarrow x^{\prime}$. The Higgs potential, invariant under CP3, has the parameters

$$
\mathrm{CP} 3: \quad \boldsymbol{\xi}=\left(\begin{array}{l}
0 \\
0 \\
0
\end{array}\right), \quad \boldsymbol{\eta}=\left(\begin{array}{l}
0 \\
0 \\
0
\end{array}\right), \quad E=\left(\begin{array}{ccc}
\mu_{1} & 0 & 0 \\
0 & \mu_{2} & 0 \\
0 & 0 & \mu_{1}
\end{array}\right) \text {. }
$$

The basis-independent formulation reads here $\boldsymbol{\xi}=0$ and $\boldsymbol{\eta}=0$, and two eigenvalues of $E$ degenerate.

The impact of the six classes on the parameters in terms of the fields is given in Table I] for a specific basis choice. The first three may be obtained from family symmetries alone. The next three may be obtained from GCP symmetries alone. The CP2 entry is presented in a very specific basis mentioned by Davidson and Haber [21. For $Z_{2}, \lambda_{5}$ may be made real without loss of generality. In appendix A we discuss cases where two symmetries are imposed simultaneously.

The models $Z_{2}, U(1), U(2), \mathrm{CP} 1, \mathrm{CP} 2$, and CP3 discussed so far obey exact symmetries. We shall also consider the case that these symmetries are broken by including all possible Higgs-potential terms of dimension two. This is known as soft breaking of the symmetry. Soft-breaking terms arise for instance if we consider a THDM as an effective model. For example, in the minimal supersymmetric extension of the Standard Model (MSSM), supersymmetry is broken by imposing soft-breaking terms. In this way mass degeneracy of the particles and their superpartners is avoided. 
TABLE I: Impact of the symmetries on the coefficients of the Higgs potential in a specific basis. See Ref. [29] for more details.

\begin{tabular}{|c|c|c|c|c|c|c|c|}
\hline symmetry $m_{11}^{2}$ & $m_{22}^{2}$ & $m_{12}^{2}$ & $\lambda_{1} \lambda_{2} \lambda_{3}$ & $\lambda_{4}$ & $\lambda_{5}$ & $\lambda_{6}$ & $\lambda_{7}$ \\
\hline$Z_{2}$ & & 0 & & & real & 0 & 0 \\
\hline$U(1)$ & & 0 & & & 0 & 0 & 0 \\
\hline$U(2)$ & $m_{11}^{2}$ & 0 & $\lambda_{1}$ & $\lambda_{1}-\lambda_{3}$ & 0 & 0 & 0 \\
\hline $\mathrm{CP} 1$ & & real & & & real & real & $\lambda_{6}$ \\
\hline $\mathrm{CP} 2$ & $m_{11}^{2}$ & 0 & $\lambda_{1}$ & & real & 0 & 0 \\
\hline CP3 & $m_{11}^{2}$ & 0 & $\lambda_{1}$ & & $\lambda_{1}-\lambda_{3}-\lambda_{4}($ real $)$ & 0 & 0 \\
\hline
\end{tabular}

Another example is the next-to-minimal supersymmetric Standard Model (NMSSM), wherein the $\mu$ term is generated by the vacuum expectation value of a singlet field. For a review of the NMSSM see, for instance, 38 . If the first symmetry breaking occurs at an energy scale $v_{s}$ considerably larger than the electroweak scale $v_{0}$, we obtain below the scale $v_{s}$ an effective THDM with soft-breaking terms [39]. This type of NMSSM model is in fact a particular example of theories containing two doublets and additional singlet fields where the latter acquire vevs and in this way produce a low-energy effective theory analogous to the softly-broken THDM's discussed here. In general, soft breaking of a symmetry is employed in order to avoid unwanted massless Goldstone modes in case the symmetry is continuous or in order to avoid the domain-wall problem in case of a discrete symmetry. A further motivation to consider soft breaking is as follows. Theories with a softly broken symmetry preserve under at least one-loop renormalization whatever relations between the quartic parameters were imposed by the exact symmetry.

In theories with general soft breaking, $m_{11}^{2}, m_{22}^{2}, \operatorname{Re}\left(m_{12}^{2}\right)$, and $\operatorname{Im}\left(m_{12}^{2}\right)$ in the potential (1.1) do not vanish and bear no relation to each other. According to (1.5), this corresponds in $K$ space to a non-vanishing vector

$$
\boldsymbol{\xi}=\left(\begin{array}{l}
\xi_{1} \\
\xi_{2} \\
\xi_{3}
\end{array}\right)
$$

Thus, in the following we shall study THDM's with softly broken $Z_{2}, \ldots$, CP3 symmetries, that is, THDM's where the parameters $\boldsymbol{\eta}$ and $E$ are for the various classes as in 2.4 , (2.7), (2.10), (2.11), (2.13), 2.14), respectively, but $\boldsymbol{\xi}$ is left arbitrary.

\section{CP PROPERTIES}

The main reason why T.D. Lee introduced the THDM in 1973 [1] was precisely because one could have spontaneous $\mathrm{CP}$ breaking in the model. In fact it is well known that spontaneous CP breaking occurs in the $Z_{2}$ model with soft breaking [36] or in the CP1 model, but that it cannot occur in the unbroken $Z_{2}$ and $U(1)$ cases; see, for instance, [4, 6].

In this section we will show that the powerful bilinear formalism, coupled with the equally powerful basis-invariant methods, allows us to investigate CP breaking, explicit or spontaneous, for all symmetry-constrained THDM Higgs potentials discussed in the last section. As we will see this is possible for the most part, even without computing the vacuum-expectation values. We must however emphasize that in the following discussion of the various models we always assume that the parameters 1.5-1.7) of the potential are chosen such that we have stability and a neutral vacuum different from $\langle\tilde{\mathbf{K}}\rangle=0$.

We note that we have obtained numerical examples with these properties for each case discussed below. Since the conditions for the CP symmetries (1.21)- 1.24$),(1.25)-(1.27)$ are basis independent, we can apply them to models given in any basis. We use this freedom to consider the models in convenient bases - for instance in bases where the parameter matrix $E$ is diagonal. Since the conditions are not affected by a change of basis the results hold for the models basis independently. It is also straightforward to formulate the results written down below in the basis where $E$ is diagonal in an explicitly basis-invariant way: clearly, the eigenvalues of $E$ are basis-invariant quantities. Furthermore, suppose that we use the components $\xi_{1}, \xi_{2}, \xi_{3}$ of the vector $\boldsymbol{\xi}$ in the $E$-diagonal basis. In a basisindependent formulation these components of $\boldsymbol{\xi}$ are the projections of $\boldsymbol{\xi}$ onto the normalized eigenvectors of $E$. The same holds for these components of the other vectors, $\boldsymbol{\eta}$ and $\langle\mathbf{K}\rangle$. In the following explicitly basis-independent formulations are always easily obtainable in this way. 


\section{The $U(2)$ model}

\section{Explicit CP breaking}

In the unbroken $U(2)$ model we have $\boldsymbol{\xi}=\boldsymbol{\eta}=0$, thus from 1.21 - follows $I_{1}=I_{2}=I_{3}=I_{4}=0$, that is, we have explicit CP conservation. Even in the soft breaking case, with $\boldsymbol{\xi}$ arbitrary, we find from $\boldsymbol{\eta}=0$ with 1.21 - (1.24) immediately that $I_{1}=I_{2}=I_{4}=0$. Moreover, since the matrix $E$ is proportional to the identity, the vector $E \boldsymbol{\xi}$ is parallel to $\boldsymbol{\xi}$ and thus, from $(1.23)$, we also obtain $I_{3}=0$, with or without soft breaking terms. We find that there is at least one explicit CP symmetry in the $U(2)$ model, even in the presence of the soft-breaking terms.

\section{Spontaneous CP breaking}

Since $\boldsymbol{\eta}=0$ we have $J_{1}=J_{3}=0$ and since $\boldsymbol{\xi}$ and $E \boldsymbol{\xi}$ vanish for the exact symmetry and are parallel in the soft-breaking case, $J_{2}$ is also zero, as can be seen from (1.25) to (1.27). Hence, at least one CP symmetry is conserved by the vacuum, even if we take the most general soft breaking terms into account.

\section{The CP3 model}

\section{Explicit CP breaking}

The main difference with regard to the $U(2)$ model is that now $E$ is not proportional to the identity - only two of the eigenvalues are degenerate. The unbroken CP3 model implies that $\boldsymbol{\eta}=\boldsymbol{\xi}=0$, so we get here $I_{1}=I_{2}=I_{3}=I_{4}=0$. Let us turn to the softly broken case with a non-vanishing vector $\boldsymbol{\xi}$. Since $\boldsymbol{\eta}=0$, we find immediately $I_{1}=I_{2}=I_{4}=0$. The invariant $I_{3}$ reads in any basis with a diagonal matrix $E$

$$
I_{3}=\xi_{1} \xi_{2} \xi_{3}\left(\mu_{1}-\mu_{2}\right)\left(\mu_{2}-\mu_{3}\right)\left(\mu_{3}-\mu_{1}\right) .
$$

From the degeneracy of two eigenvalues of $E$ follows that $I_{3}=0$. We see this also directly from 1.23 . Because $E$ has two degenerate eigenvalues the vectors $\boldsymbol{\xi}, E \boldsymbol{\xi}, E^{2} \boldsymbol{\xi}$ lie in one plane, that is, the triple product in $I_{3}$ vanishes. Thus, the CP3 model is explicitly CP conserving, even in the softly broken case [42].

\section{Spontaneous CP breaking}

In the unbroken model we have $\boldsymbol{\eta}=\boldsymbol{\xi}=0$ and get immediately from (1.25) to 1.27) that all $J^{\prime} s$ are zero. In the softly broken case, with $\boldsymbol{\xi}$ arbitrary, we still have $\boldsymbol{\eta}=0$, that is, $J_{1}$ and $J_{3}$ are zero. Since $\mu_{1}=\mu_{3}$, we may perform a basis transformation such that $\xi_{3}=0$, leaving $\boldsymbol{\eta}$ and $E$ unchanged. With this simplification, $J_{2}$ is given by

$$
J_{2}=\xi_{1} \xi_{2}\left(\mu_{2}-\mu_{1}\right)\left\langle K_{3}\right\rangle .
$$

In order to derive further conclusions we look at the stationarity conditions 1.30 . We get explicitly

$$
\left(\begin{array}{c}
\left\langle K_{0}\right\rangle\left(\eta_{00}-u\right) \\
\left\langle K_{1}\right\rangle\left(\mu_{1}+u\right) \\
\left\langle K_{2}\right\rangle\left(\mu_{2}+u\right) \\
\left\langle K_{3}\right\rangle\left(\mu_{1}+u\right)
\end{array}\right)=-\frac{1}{2}\left(\begin{array}{c}
\xi_{0} \\
\xi_{1} \\
\xi_{2} \\
0
\end{array}\right) .
$$

For the case $\mu_{1}+u \neq 0$ there is only a solution for $\left\langle K_{3}\right\rangle=0$. On the other hand, for $\mu_{1}+u=0$, we get $\xi_{1}=0$. Thus we see from (3.2) that in either case the condition $J_{2}=0$ is fulfilled. Hence, for the unbroken and softly broken CP3 model, there is at least one CP symmetry respected by the vacuum.

\section{The $U(1)$ model}

Explicit CP breaking

Consider first the exactly symmetric model with parameters (2.7). The vectors $\boldsymbol{\eta}$ and $\boldsymbol{\xi}$ are not zero, but they are parallel to each other, as are $E \boldsymbol{\eta}$ and $E \boldsymbol{\xi}$. This means that all the $I^{\prime} s$ are zero, as is easy to see from (1.21)-1.24. 
In the softly broken case, since we have two degenerate eigenvalues in the matrix $E$, we find that still all $I^{\prime} s$ vanish; see (3.1). Therefore, we have explicit CP conservation with the $U(1)$ scalar potential, even if we take soft breaking terms into account.

\section{Spontaneous CP breaking}

For the unbroken model, since $\boldsymbol{\eta}, \boldsymbol{\xi}, E \boldsymbol{\xi}, E \boldsymbol{\eta}$ are parallel, all $J^{\prime} s$ are zero. For the softly broken $U(1)$ case we can, without loss of generality, go into a basis with $\xi_{2}=0$. We get then for the $J^{\prime} s$ from (1.25) to (1.27)

$$
J_{1}=-\xi_{1} \eta_{3}\left\langle K_{2}\right\rangle, \quad J_{2}=\xi_{1} \xi_{3}\left(\mu_{1}-\mu_{3}\right)\left\langle K_{2}\right\rangle, \quad J_{3}=0 .
$$

Therefore, we need to look at the vacuum properties before we can reach further conclusions. Employing again the stationarity conditions 1.30 , we get

$$
\left(\begin{array}{c}
\left\langle K_{0}\right\rangle\left(\eta_{00}-u\right)+\left\langle K_{3}\right\rangle \eta_{3} \\
\left\langle K_{1}\right\rangle\left(\mu_{1}+u\right) \\
\left\langle K_{2}\right\rangle\left(\mu_{1}+u\right) \\
\left\langle K_{3}\right\rangle\left(\mu_{3}+u\right)+\left\langle K_{0}\right\rangle \eta_{3}
\end{array}\right)=-\frac{1}{2}\left(\begin{array}{c}
\xi_{0} \\
\xi_{1} \\
0 \\
\xi_{3}
\end{array}\right) .
$$

This means that we get for $\mu_{1}+u \neq 0$ directly $\left\langle K_{2}\right\rangle=0$ and for $\mu_{1}+u=0$ we have $\xi_{1}=0$. We see from (3.4), that in either case all $J^{\prime} s$ are zero and we have at least one CP symmetry which is preserved by the vacuum.

\section{The CP2 model}

\section{Explicit CP breaking}

Here, for the unbroken case, $\boldsymbol{\eta}=\boldsymbol{\xi}=0$ implies again that all the $I$ 's are zero, that is, we have explicit CP conservation, as trivially expected for a $\mathrm{CP}$ invariant model. In the softly broken case, with an arbitrary vector $\boldsymbol{\xi}$, only $I_{3}$ as given in (3.1) may be non-zero. In the case of the softly broken CP2 model with non-degenerate eigenvalues of $E$, we thus in general do not have explicit $\mathrm{CP}$ conservation.

However, in the softly-broken CP2 model it is still possible to choose the soft-breaking parameters such that CP is explicitly conserved. For instance, in the case that at least one of the components of $\boldsymbol{\xi}$ is zero (in a basis where $E$ is diagonal) we have explicit CP invariance. It is interesting to consider a few particular cases: if $\xi_{1}=\xi_{2}=0$ (and $\left.\xi_{3} \neq 0\right)$, corresponding to $\operatorname{Re}\left(m_{12}^{2}\right)=\operatorname{Im}\left(m_{12}^{2}\right)=0$, then we see from a comparison of 2.13) with 2.4 or from Table I that the softly-broken CP2 model has a remaining $Z_{2}$ symmetry $\left(\varphi_{1} \rightarrow \varphi_{1} ; \varphi_{2} \rightarrow-\varphi_{2}\right)$. Similarly, if $\xi_{2}=\xi_{3}=0$ (and $\left.\xi_{1} \neq 0\right)$, corresponding to $\operatorname{Im}\left(m_{12}^{2}\right)=m_{22}^{2}-m_{11}^{2}=0$, then the softly-broken CP2 model has a remaining $\Pi_{2}$ symmetry $\left(\varphi_{1} \leftrightarrow \varphi_{2}\right.$, which is simply $Z_{2}$ in a different basis; see appendix A). Furthermore, if we choose $\xi_{2}=0\left(\operatorname{Im}\left(m_{12}^{2}\right)=0\right)$ we see from (3.1) that the model will have an unbroken CP symmetry. Note that the cases of degenerate eigenvalues in the matrix $E$ correspond actually to the CP3 model (maybe in a different basis) or to the $U(2)$ model. All of these symmetries $-Z_{2}, \Pi_{2}$, and CP1 - if exact, imply explicit CP conservation.

\section{Spontaneous CP breaking}

For the unbroken case, $\boldsymbol{\eta}=\boldsymbol{\xi}=0$, we see from (1.25) to 1.27 that all $J$ 's are trivially zero, that is we have spontaneous $\mathrm{CP}$ conservation. For the softly broken model, $\boldsymbol{\xi} \neq 0$, consider the case of explicit CP conservation, that is, the case with all $I^{\prime} s$, in particular $I_{3}$ in (3.1), vanishing. We have then $J_{1}=J_{3}=0$, and $J_{2}$ given by

$$
J_{2}=\xi_{2} \xi_{3}\left(\mu_{3}-\mu_{2}\right)\left\langle K_{1}\right\rangle+\xi_{1} \xi_{3}\left(\mu_{1}-\mu_{3}\right)\left\langle K_{2}\right\rangle+\xi_{1} \xi_{2}\left(\mu_{2}-\mu_{1}\right)\left\langle K_{3}\right\rangle .
$$

In order to reach further conclusions about the spontaneous breaking behavior we consider the stationarity conditions 1.30,

$$
\left(\begin{array}{c}
\left\langle K_{0}\right\rangle\left(\eta_{00}-u\right) \\
\left\langle K_{1}\right\rangle\left(\mu_{1}+u\right) \\
\left\langle K_{2}\right\rangle\left(\mu_{2}+u\right) \\
\left\langle K_{3}\right\rangle\left(\mu_{3}+u\right)
\end{array}\right)=-\frac{1}{2}\left(\begin{array}{c}
\xi_{0} \\
\xi_{1} \\
\xi_{2} \\
\xi_{3}
\end{array}\right)
$$


We see that for a general solution of the stationarity conditions we do not necessarily find $J_{2}=0$ in the soft breaking case, that is, we have in general spontaneous $\mathrm{CP}$ violation.

Remember that it is sufficient to have one of the components of $\boldsymbol{\xi}$ equal to zero in order to have explicit CP conservation in the CP2 model. For instance, if $\xi_{2}=0$ and $u \neq-\mu_{2}$, then $\left\langle K_{2}\right\rangle=0$ and $J_{2}=0$. But the solution $u=-\mu_{2}$ and $\left\langle K_{2}\right\rangle \neq 0$ is also a possibility, and this corresponds to a vacuum with spontaneous CP violation.

To summarize: a model with exact CP2 symmetry is explicitly and spontaneously CP conserving, that is, at least one CP symmetry is conserved explicitly and by the vacuum. This was already proven in [11. For the softly broken case the parameters can be chosen such as to have explicit CP conservation. For the case of explicit CP conservation we may have spontaneous $\mathrm{CP}$ violation.

The $Z_{2}$ model

\section{Explicit CP breaking}

This case is quite similar to that of the $U(1)$ model: for the unbroken model the vectors $\boldsymbol{\eta}, \boldsymbol{\xi}, E \boldsymbol{\eta}$ and $E \boldsymbol{\xi}$ are all parallel to each other and, therefore, the $I$ invariants are zero. For the softly broken case we still have $I_{2}=I_{4}=0$, $I_{3}$ is given in (3.1), and

$$
I_{1}=\xi_{1} \xi_{2} \eta_{3}\left(\mu_{1}-\mu_{2}\right) .
$$

Thus, in general the softly broken $Z_{2}$ model is explicitly $\mathrm{CP}$ violating. But, obviously, in particular cases it is possible to have explicit CP conservation. For instance, for the case that one of the parameters $\xi_{1}$ or $\xi_{2}$ is equal to zero, or that the two eigenvalues $\mu_{1}$ and $\mu_{2}$ of the matrix $E$ are degenerate, we find from (3.1) and (3.8) that all $I^{\prime} s$ vanish. But one must study whether that reproduces one of the other five symmetry-constrained models. For example, starting from the softly-broken $Z_{2}$ model and imposing $\mu_{1}=\mu_{2}$ we recover the explicitly CP conserving softly-broken $U(1)$ model. Likewise, for $\eta_{3}=0$ we end up with the softly broken CP2 model. Thus, in studying the $Z_{2}$ model with soft breaking and explicit CP conservation we may focus on the cases where either $\xi_{1}$ or $\xi_{2}$ is zero.

\section{Spontaneous CP breaking}

For the unbroken $Z_{2}$ model, the vectors $\boldsymbol{\xi}, \boldsymbol{\eta}, E \boldsymbol{\xi}, E \boldsymbol{\eta}$ entering into the computation of the $J$ invariants in 1.25 1.27 are parallel. Thus, all $J^{\prime} s$ vanish. The exact $Z_{2}$ symmetry implies spontaneous CP conservation, as is well known; see for example [4, 6, 36.

Concerning the softly broken $Z_{2}$ model suppose that we have explicit CP conservation, that is, the parameters are such that besides $I_{2}$ and $I_{4}$, also $I_{3}(3.1)$ and $I_{1}(3.8)$ vanish. As already discussed, we can disregard the cases $\mu_{1}=\mu_{2}$ and $\eta_{3}=0$ because they correspond to the $U(1)$ and CP2 models. Therefore, we must have $\xi_{1}=0$ or $\xi_{2}=0$. We find then $J_{3}=0, J_{2}$ is given by (3.6) and

$$
J_{1}=\eta_{3}\left(\xi_{2}\left\langle K_{1}\right\rangle-\xi_{1}\left\langle K_{2}\right\rangle\right) .
$$

The vacuum solution is determined by

$$
\left(\begin{array}{c}
\left\langle K_{0}\right\rangle\left(\eta_{00}-u\right)+\left\langle K_{3}\right\rangle \eta_{3} \\
\left\langle K_{1}\right\rangle\left(\mu_{1}+u\right) \\
\left\langle K_{2}\right\rangle\left(\mu_{2}+u\right) \\
\left\langle K_{3}\right\rangle\left(\mu_{3}+u\right)+\left\langle K_{0}\right\rangle \eta_{3}
\end{array}\right)=-\frac{1}{2}\left(\begin{array}{l}
\xi_{0} \\
\xi_{1} \\
\xi_{2} \\
\xi_{3}
\end{array}\right) .
$$

We see that even in the case where either $\xi_{1}$ or $\xi_{2}$ is equal to zero, we still have that $J_{1}$ and $J_{2}$ are not necessarily zero. For instance, for $\xi_{2}=0, \xi_{1} \neq 0$, and $u=-\mu_{2}$, we can obtain $\left\langle K_{2}\right\rangle \neq 0$, that is, $J_{1} \neq 0$. Thus, spontaneous CP violation is possible in softly broken $Z_{2}$ models, even though it cannot occur in the case of the exact symmetry. This result is well-known in fact and was shown in [36, where a $\Pi_{2}$ symmetry was considered, which is just the $Z_{2}$ model in a different basis. 


\section{The CP1 model}

Explicit CP breaking

The unbroken CP1 model has vanishing $I^{\prime} s$. It is, by construction, explicitly CP conserving. The softly broken CP1 model arises from the unbroken case by having $\xi_{2} \neq 0$. We can in addition assume that both $\eta_{1} \neq 0$ and $\eta_{3} \neq 0$, since otherwise we get the $Z_{2}$ case (or the $\Pi_{2}$ case, that is, $Z_{2}$ in a different basis). The explicit CP conditions (1.21)-(1.24) read then $I_{4}=0$,

$$
\begin{aligned}
& I_{1}=\xi_{2}\left[\xi_{1} \eta_{3}\left(\mu_{1}-\mu_{2}\right)+\xi_{3} \eta_{1}\left(\mu_{2}-\mu_{3}\right)\right]=0, \\
& I_{2}=\xi_{2} \eta_{1} \eta_{3}\left(\mu_{1}-\mu_{3}\right)=0, \\
& I_{3}=\xi_{1} \xi_{2} \xi_{3}\left(\mu_{1}-\mu_{2}\right)\left(\mu_{2}-\mu_{3}\right)\left(\mu_{3}-\mu_{1}\right)=0 .
\end{aligned}
$$

Thus, in general, we will have explicit CP violation in the softly broken CP1 model.

Spontaneous CP breaking

Suppose that CP is explicitly conserved. According to the discussion above this implies that we have the unbroken CP1 model where $\xi_{2}=0$. The $J$ invariants have then the form

$$
\begin{aligned}
& J_{1}=\left(\xi_{3} \eta_{1}-\xi_{1} \eta_{3}\right)\left\langle K_{2}\right\rangle, \\
& J_{2}=\xi_{1} \xi_{3}\left(\mu_{1}-\mu_{3}\right)\left\langle K_{2}\right\rangle, \\
& J_{3}=\eta_{1} \eta_{3}\left(\mu_{1}-\mu_{3}\right)\left\langle K_{2}\right\rangle .
\end{aligned}
$$

The stationarity conditions 1.30 are

$$
\left(\begin{array}{c}
\left\langle K_{0}\right\rangle\left(\eta_{00}-u\right)+\left\langle K_{1}\right\rangle \eta_{1}+\left\langle K_{3}\right\rangle \eta_{3} \\
\left\langle K_{1}\right\rangle\left(\mu_{1}+u\right)+\left\langle K_{0}\right\rangle \eta_{1} \\
\left\langle K_{2}\right\rangle\left(\mu_{2}+u\right) \\
\left\langle K_{3}\right\rangle\left(\mu_{3}+u\right)+\left\langle K_{0}\right\rangle \eta_{3}
\end{array}\right)=-\frac{1}{2}\left(\begin{array}{c}
\xi_{0} \\
\xi_{1} \\
0 \\
\xi_{3}
\end{array}\right) .
$$

We see that a valid vacuum may occur with $\left\langle K_{2}\right\rangle \neq 0$ with a Lagrange multiplier $u=-\mu_{2}$. Then, the three $J^{\prime} s$ do in general not vanish. Thus, the CP1 model may have spontaneous CP violation. This result was obtained by T. D. Lee a long time ago [1].

\section{Summary}

We present an overview of the CP properties of the six symmetry-constrained THDM's, with and without soft symmetry breaking, in Table II] which is the main result of this article. Table II displays the whole gamut of possibilities of CP breaking in any of the six possible classes of symmetry-constrained THDM's. As we have seen, it was possible to obtain these results without the need to determine explicit expressions for the vacuum-expectation values. Regarding the last two columns, please be aware that any model which can display explicit CP breaking therein listed can also, through a judicious choice of the parameters, explicitly preserve $\mathrm{CP}$ - and, in that case, develop vacua which spontaneously break $\mathrm{CP}$, which is indicated in the last column.

\section{CONCLUSIONS}

In this work we have performed a thorough study of the CP breaking properties, both explicit and spontaneous, of all possible THDM scalar potentials corresponding to any of the six symmetry classes. We also considered the softly broken models. Our study was performed in a very efficient manner using basis-invariant quantities written in the bilinear formalism, which simplified the analysis immensely. The results are presented in Table II This includes some results scattered through the literature, sometimes only implicitly, as well as our new results. Table II allows us to draw some general conclusions: 
TABLE II: CP properties of the six symmetry-constrained THDM's, with and without soft-symmetry breaking. "Yes" means that it is possible to choose the parameters of the potential such as to enable that particular form of CP violation.

\begin{tabular}{|c|c|c|c|c|}
\hline \hline \multirow{2}{*}{ symmetry } & \multicolumn{2}{|c|}{ exact } & \multicolumn{2}{c|}{ softly-broken } \\
\cline { 2 - 5 } & explicit & cPpontaneous & explicit & spontaneous \\
CPV & CPV & CPV \\
\hline$Z_{2}$ & - & - & Yes & Yes \\
$U(1)$ & - & - & - & - \\
$U(2)$ & - & - & - & - \\
\hline $\mathrm{CP} 1$ & - & Yes & Yes & Yes \\
$\mathrm{CP} 2$ & - & - & Yes & Yes \\
$\mathrm{CP} 3$ & - & - & - & - \\
\hline \hline
\end{tabular}

- Any exact symmetry prevents explicit $\mathrm{CP}$ breaking.

- All the models which possess a continuous symmetry - U(1), $U(2)$, and CP3 - cannot have explicit or spontaneous CP breaking, even with the most general soft breaking terms.

- All models with discrete symmetries $-Z_{2}, \mathrm{CP} 1$, and $\mathrm{CP} 2$ - may have explicit $\mathrm{CP}$ violation, but they require soft symmetry breaking terms to make it happen.

- In models with exact symmetries $Z_{2}$ or $\mathrm{CP} 2$ there is no spontaneous CP violation. But we remind the reader that this only means that at least one CP symmetry is preserved by the vacuum. The unbroken CP2 model has four GCP symmetries where two are broken and two are unbroken if the theory has the correct EWSB; see [11].

- In the models with softly broken discrete symmetries, $Z_{2}$ or $\mathrm{CP} 2$, we may choose the parameters in a way to achieve explicit CP conservation. Then these models may have spontaneous $\mathrm{CP}$ violation.

- In models with exact CP1 symmetry, spontaneous CP violation is possible as has been known for a long time 1 . Inclusion of soft-breaking terms either violates the CP symmetry explicitly or leads back to the exactly symmetric case.

We remind the reader that our analysis concerned only the scalar sector, nothing was said about CP violation in the Yukawa sector. There one can have explicit CP violation even if the scalar potential is explicitly CP conserving (the SM is a prime example of this). Also, a Lagrangian where the Higgs potential preserves CP explicitly and spontaneously may well break CP spontaneously in the fermion sector. An example of this unusual phenomenon was found in [37].

\section{Acknowledgments}

The work of P.M.F. is supported in part by the Portuguese Fundação para a Ciência e a Tecnologia (FCT) under contract PTDC/FIS/70156/2006. The work of J.P.S. is funded by FCT through the projects CERN/FP/109305/2009 and U777-Plurianual, and by the EU RTN project Marie Curie: MRTN-CT-2006-035505. The work of M.M. was funded by Deutsche Forschungsgemeinschaft, project number NA296/5-1. P.F. thanks the gracious hospitality of the Institut für Theoretische Physik at Heidelberg during part of this work. M.M. thanks the Centro de Física Teórica e Computacional at Universidade de Lisboa for its hospitality during his visit to Lisbon.

\section{Appendix A: Multiple Higgs-family symmetries}

In 29] it was shown that the simultaneous imposition of two Higgs-family symmetries corresponds in a certain basis to another Higgs-family symmetry in some cases. Here we want to show these results and similar ones in terms of the bilinear formalism [8, 11]. But let us first recall the $\Pi_{2}$ transformation

$$
\varphi_{1} \leftrightarrow \varphi_{2}
$$


which corresponds in $K$ space to a rotation by $\pi$ around the first axis

$$
\left(\begin{array}{l}
K_{1} \\
K_{2} \\
K_{3}
\end{array}\right) \rightarrow\left(\begin{array}{ccc}
1 & 0 & 0 \\
0 & -1 & 0 \\
0 & 0 & -1
\end{array}\right)\left(\begin{array}{l}
K_{1} \\
K_{2} \\
K_{3}
\end{array}\right)
$$

Clearly, by a basis change $\Pi_{2}$ is equivalent to $Z_{2},(2.2)$.

Now we discuss some cases of multiple symmetries:

- Simultaneous $U(1)$ and CP3 symmetries. From (2.7) and (2.14) we see, that in the basis chosen there both symmetries combined give the $U(2)$ model 2.10 . Thus, we confirm that in a certain basis $U(1) \oplus \mathrm{CP} 3=U(2)$.

- Next we introduce the $\Pi_{2}$ symmetric model, which, by a basis change, is equivalent to $Z_{2}$ :

$$
\Pi_{2}: \quad \boldsymbol{\xi}=\left(\begin{array}{c}
\xi_{1} \\
0 \\
0
\end{array}\right), \quad \boldsymbol{\eta}=\left(\begin{array}{c}
\eta_{1} \\
0 \\
0
\end{array}\right), \quad E=\left(\begin{array}{ccc}
\mu_{1} & 0 & 0 \\
0 & \mu_{2} & 0 \\
0 & 0 & \mu_{3}
\end{array}\right) .
$$

Applying simultaneously $U(1)$ and $\Pi_{2}$ we get

$$
\boldsymbol{\xi}=\left(\begin{array}{l}
0 \\
0 \\
0
\end{array}\right), \quad \boldsymbol{\eta}=\left(\begin{array}{l}
0 \\
0 \\
0
\end{array}\right), \quad E=\left(\begin{array}{ccc}
\mu_{1} & 0 & 0 \\
0 & \mu_{1} & 0 \\
0 & 0 & \mu_{3}
\end{array}\right) .
$$

This is, by a basis change, the CP3 model 2.14). We thus confirm $U(1) \oplus \Pi_{2}=$ CP3 in a certain basis.

- Applying simultaneously $Z_{2}$ (2.4) and $\Pi_{2}$ (A.3) we get immediately CP2 2.13). That is we confirm $Z_{2} \oplus \Pi_{2}=$ CP2 in a certain basis.

In a similar way we can show that $Z_{2} \oplus \mathrm{CP} 1=\mathrm{CP} 2, U(1) \oplus \mathrm{CP} 1=\mathrm{CP} 3, U(2) \oplus \mathrm{CP} 2=\mathrm{CP} 3, U(1) \oplus U(1)^{\prime}=U(2)$, and $\mathrm{CP} 3 \oplus \mathrm{CP}^{\prime}=U(2)$ in certain bases.

[1] T. D. Lee, "A Theory of Spontaneous T Violation," Phys. Rev. D 8, 1226 (1973).

[2] J. F. Gunion, H. E. Haber, G. L. Kane and S. Dawson, "The Higgs Hunter's Guide" (Perseus Publishing, Cambridge, MA, 1990).

[3] J. F. Gunion, H. E. Haber, G. L. Kane and S. Dawson, "Errata for the Higgs hunter's guide", arXiv:hep-ph/9302272.

[4] J. Velhinho, R. Santos and A. Barroso, "Tree level vacuum stability in two-Higgs doublet models," Phys. Lett. B 322, 213 (1994).

[5] F. Nagel, "New aspects of gauge-boson couplings and the Higgs sector", phD thesis, (2004), http://www.slac.stanford. edu/spires/find/hep/www?irn=6461018

[6] P. M. Ferreira, R. Santos and A. Barroso, "Stability of the tree-level vacuum in two Higgs doublet models against charge or CP spontaneous violation", Phys. Lett. B 603, 219 (2004) [Erratum-ibid. B 629, 114 (2005)] arXiv:hep-ph/0406231].

[7] I. P. Ivanov, "Two-Higgs-doublet model from the group-theoretic perspective", Phys. Lett. B 632, 360 (2006) arXiv:hep-ph/0507132.

[8] M. Maniatis, A. von Manteuffel, O. Nachtmann and F. Nagel, "Stability and symmetry breaking in the general two-Higgsdoublet model", Eur. Phys. J. C 48 (2006) 805 arXiv:hep-ph/0605184.

[9] C. C. Nishi, "CP violation conditions in N-Higgs-doublet potentials", Phys. Rev. D 74, 036003 (2006) [Erratum-ibid. D 76, 119901 (2007)] arXiv:hep-ph/0605153.

[10] I. P. Ivanov, "Minkowski space structure of the Higgs potential in 2HDM", Phys. Rev. D 75, 035001 (2007) [Erratum-ibid. D 76, 039902 (2007)] arXiv:hep-ph/0609018].

[11] M. Maniatis, A. von Manteuffel and O. Nachtmann, "CP Violation in the General Two-Higgs-Doublet Model: a Geometric View", Eur. Phys. J. C 57 (2008) 719 arXiv:0707.3344 [hep-ph]].

[12] C. C. Nishi, "The structure of potentials with N Higgs doublets", Phys. Rev. D 76, 055013 (2007) arXiv:0706.2685 [hep-ph]].

[13] I. P. Ivanov, "Minkowski space structure of the Higgs potential in 2HDM: II. Minima, symmetries, and topology", Phys. Rev. D 77, 015017 (2008) arXiv:0710.3490 [hep-ph]].

[14] C. C. Nishi, "Physical parameters and basis transformations in the Two-Higgs-Doublet model", Phys. Rev. D 77, 055009 (2008) arXiv:0712.4260 [hep-ph]]. 
[15] M. Maniatis, A. von Manteuffel and O. Nachtmann, "Determining the global minimum of Higgs potentials via Groebner bases - applied to the NMSSM", Eur. Phys. J. C 49 (2007) 1067 arXiv:hep-ph/0608314.

[16] L. Lavoura and J. P. Silva, "Fundamental CP violating quantities in a SU(2) x U(1) model with many Higgs doublets," Phys. Rev. D 50, 4619 (1994) arXiv:hep-ph/9404276.

[17] F. J. Botella and J. P. Silva, "Jarlskog - like invariants for theories with scalars and fermions," Phys. Rev. D 51, 3870 (1995) arXiv:hep-ph/9411288.

[18] J. F. Gunion, talk given at the CPNSH, CERN, Switzerland, December (2004).

[19] I. F. Ginzburg and M. Krawczyk, "Symmetries of two Higgs doublet model and CP violation," Phys. Rev. D 72, 115013 (2005) arXiv:hep-ph/0408011.

[20] G. C. Branco, M. N. Rebelo and J. I. Silva-Marcos, "CP-odd invariants in models with several Higgs doublets," Phys. Lett. B 614, 187 (2005) arXiv:hep-ph/0502118.

[21] S. Davidson and H. E. Haber, "Basis-independent methods for the two-Higgs-doublet model," Phys. Rev. D 72, 035004 (2005) [Erratum-ibid. D 72, 099902 (2005)] arXiv:hep-ph/0504050.

[22] J. F. Gunion and H. E. Haber, "Conditions for CP-violation in the general two-Higgs-doublet model," Phys. Rev. D 72, 095002 (2005) arXiv:hep-ph/0506227.

[23] T. D. Lee and G. C. Wick, "Space inversion, time reversal, and other discrete symmetries in local field theories", Phys. Rev. 148, 1385 (1966).

[24] G. Ecker, W. Grimus and W. Konetschny, "Quark Mass Matrices In Left-Right Symmetric Gauge Theories," Nucl. Phys. B 191 (1981) 465,

[25] G. Ecker, W. Grimus and H. Neufeld, "Spontaneous CP Violation In Left-Right Symmetric Gauge Theories," Nucl. Phys. B 247 (1984) 70,

[26] J. Bernabeu, G. C. Branco and M. Gronau, "CP Restrictions On Quark Mass Matrices," Phys. Lett. B 169, 243 (1986).

[27] G. Ecker, W. Grimus and H. Neufeld, "A Standard Form For Generalized CP Transformations," J. Phys. A 20 (1987) L807.

[28] H. Neufeld, W. Grimus and G. Ecker, "Generalized CP invariance, neutral flavor conservation and the structure of the mixing matrix", Int. J. Mod. Phys. A 3 (1988) 603.

[29] P. M. Ferreira, H. E. Haber and J. P. Silva, "Generalized CP symmetries and special regions of parameter space in the two-Higgs-doublet model," Phys. Rev. D 79, 116004 (2009) arXiv:0902.1537 [hep-ph]].

[30] M. Maniatis, A. von Manteuffel and O. Nachtmann, "A new type of CP symmetry, family replication and fermion mass hierarchies", Eur. Phys. J. C 57, 739 (2008) arXiv:0711.3760 [hep-ph]].

[31] S. L. Glashow and S. Weinberg, "Natural Conservation Laws For Neutral Currents" Phys. Rev. D 151958 (1977).

[32] E. A. Paschos, "Diagonal Neutral Currents" Phys. Rev. D 15, 1966 (1977).

[33] P. Fayet, "Supergauge Invariant Extension Of The Higgs Mechanism And A Model For The Electron And Its Neutrino," Nucl. Phys. B 90, 104 (1975).

[34] R. D. Peccei and H. R. Quinn, "CP Conservation In The Presence Of Instantons," Phys. Rev. Lett. 38, 1440 (1977).

[35] R. D. Peccei and H. R. Quinn, "Constraints Imposed By CP Conservation In The Presence Of Instantons," Phys. Rev. D 16, 1791 (1977).

[36] G. C. Branco and M. N. Rebelo, "The Higgs Mass In A Model With Two Scalar Doublets And Spontaneous CP Violation," Phys. Lett. B 160, 117 (1985).

[37] P. M. Ferreira and J. P. Silva, "A Two-Higgs Doublet Model With Remarkable CP Properties," arXiv:1001.0574 [hep-ph]], to appear in Eur. Phys. J. C.

[38] M. Maniatis, "The Next-to-minimal supersymmetric extension of the Standard Model reviewed," Int. J. Mod. Phys. A 25 3505 (2010) arXiv:0906.0777 [hep-ph]].

[39] E. Ma and M. Maniatis, "Effective Two Higgs Doublets in Nonminimal Supersymmetric Models," arXiv:1005.3305 [hep-ph].

[40] Our convention for the $I^{\prime} s$ is equivalent to but differs from those adopted in [9, 18, 22.

[41] Our convention for the $J^{\prime} s$ follows [11] and is equivalent to but differs from that adopted in [16, 17].

[42] In this paper we are concerned only with the scalar potential. However, should one include the fermionic sector, it has been proven that the softly broken CP3 model has strange CP violating properties 37. 